Can Monetary Policy Delay the Reallocation of Capital?

Fabian Schnell

October 2013 Discussion Paper no. 2013-29 


$\begin{array}{ll}\text { Editor: } & \text { Martina Flockerzi } \\ & \text { University of St.Gallen } \\ & \text { School of Economics and Political Science } \\ & \text { Department of Economics } \\ & \text { Bodanstrasse 8 } \\ & \text { CH-9000 St. Gallen } \\ & \text { Phone } \quad+41712242325 \\ & \text { Fax } \quad+41712243135 \\ & \text { Email seps@unisg.ch } \\ & \text { School of Economics and Political Science } \\ & \text { Department of Economics } \\ & \text { University of St.Gallen } \\ & \text { Bodanstrasse } 8 \\ \text { Publisher: } & \text { CH-9000 St. Gallen } \\ & \text { Phone +41 71 224 23 25 } \\ & \text { Fax }+41712243135 \\ & \text { http://www.seps.unisg.ch }\end{array}$




\title{
Can Monetary Policy Delay the Reallocation of Capital? ${ }^{1}$
}

\author{
Fabian Schnell ${ }^{2}$
}

Author's address:

Fabian Schnell, M.A. HSG

Swiss Institute for International Economics and Applied

Economic Research

Bodanstrasse 8

$\mathrm{CH}-9000$ St. Gallen

Phone $\quad+41712242375$

Email fabian.schnell@unisg.ch

Website www.siaw.unisg.ch

\footnotetext{
${ }^{1}$ The author thanks Reto Foellmi, Johannes Fritz, Jürg Müller, Frédéric Pittet, Rafael Plessow, Lukas Schmid, Darius Stuker, and the participants of the 2012 congress of the Swiss Society of Economics and Statistics, the May 24, 2012 Sinergia seminar at the University of Zurich, and the 2013 „Structural Change, Dynamics, and Economics Growth" conference in Livorno for helpful comments. All remaining errors are mine. The views expressed in this paper are those of the author and do not necessarily represent those of economiesuisse. Financial support by the Swiss National Science Foundation is gratefully acknowledged.

${ }^{2}$ University of St. Gallen and economiesuisse.
} 


\begin{abstract}
This paper examines the medium-run effects of monetary policy and focuses its analyses on the consequences of distorted (in the sense of exogenously influenced) real interest rates that are currently observed in many industrialized countries. In our model, real interest rates that are too low hinder economic recovery because such rates allow relatively unproductive firms to remain in the market. Monetary policy should increase interest rates after a negative macroeconomic shock to force a reallocation of production factors to more productive firms. We show that there is a trade-off between the short-run and medium-run preferences of the central bank as a consequence. From a welfare perspective, the impact of monetary policy depends on the long-run interest rate relative to the welfare-maximizing interest rate because of the preference for variety in the model.
\end{abstract}

\title{
Keywords
}

Monetary Policy Design, Reallocation of Capital, Structural Change, Heterogeneous Firms.

\section{JEL Classification}

E32, E43, E50, E52. 


\section{Introduction}

After the recent financial crisis, monetary authorities around the world resolutely intervened and have thus far prevented the world economy from suffering through a second Great Depression (as was experienced in the 1930s). Nevertheless, it is sobering that the United States, the countries in the Euro Zone, and other industrialized economies are in their sixth year of less-than-potential growth. A quick turnaround is not in sight. Central banks have reacted to these ongoing recessional tendencies with more monetary stimuli as part of an unconventional monetary policy (i.e., "quantitative easing"). Data on interest rates show that at least the United States Federal Reserve (FED) has been successful with its policy regarding its impact on interest rates. Figure 1 depicts the development of real interest rates for several maturities (illustrated by inflation-protected T-Bills) in the United States between 2003 and 2012, which reveals a continuous and sustainable decrease in the real interest rate for all maturities considered. ${ }^{1}$ Thus, central banks seem to be able to influence real interest rates to the extent that they are not lower for real, structural reasons.

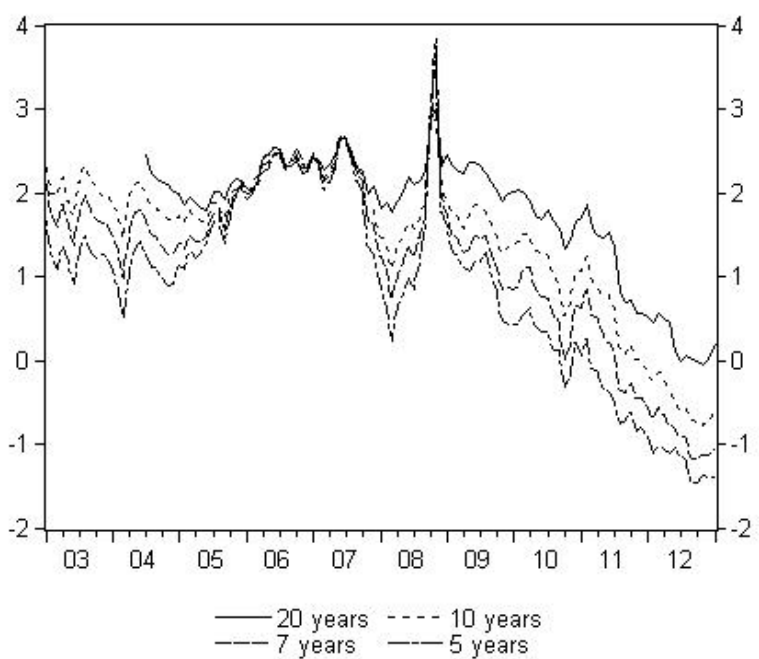

Figure 1: Real interest rates in the United States (inflation-protected T-Bills) since 2003 for 20-, 10-, 7-, and 5-year maturities. Source: U.S. Department of the Treasury.

\footnotetext{
${ }^{1}$ This impression is supported by empirical evidence. In a regression of the real interest rate on its lag, a constant, and the official federal funds rate, a Quandt-Andrews test indicates that there is a structural break in the constant for the entire sample, whereas it does not demonstrate a similar finding for a time-restricted pre-crisis sample (the latter result is consistent with Gerlach and Moretti (2011)). This implies a statistically significant decrease in real interest rates. See Appendix A for the detailed results of the econometric analysis.
} 
This paper analyses the potential effects of "distort" real interest rates in a simple industrial model with heterogeneous firms, as developed by Hopenhayn (1992) and advanced by Melitz (2003). Both models assume heterogeneous productivity. Whereas Melitz (2003) focuses on the implications of trade on the structure of an economy, we consider the influence of monetary policy on the allocation of capital in an economy through its impact on firms' cost structures. Increasing interest rates make the cost of borrowing too expensive for relatively unproductive firms, which forces them to exit the market. Thus, the resources of these firms can be reallocated to more productive uses. Higher interest rates therefore increase average productivity and quantitative output but reduce variety, implying that there is a welfare-maximizing interest rate that takes into account households' preference for variety.

We assume that central banks are able to influence the allocation of capital in the economy (and therefore its medium-term development) through monetary policy. Indeed, the model suggests that the extent of the reaction of a central bank to fluctuations (regarding price level and output stability) has an impact on output and price level. This impact is shown to be inverse in the medium-run economy compared to the classic short-run influence that is typically considered for central banks; a stabilizing monetary policy after a long-lasting negative shock must increase interest rates in the mediumrun economy when output is below its natural level, and vice versa. ${ }^{2}$ Thus, our model suggests a trade-off between short-term and medium-term goals for a central bank.

This important observation stems from the tasks and measures typically faced by central banks in nearly all industrialized countries: stabilizing consumer prices and dampening the effects of business cycles, particularly of recessions. ${ }^{3}$ To fulfill those goals, it is common wisdom that interest rates are lowered in a recession, stimulating investment and helping economic development recover. Conversely, when fighting inflation and stabilizing prices, central banks reduce the money supply, which implies increasing interest rates. Nevertheless, this situation might only hold for the short-run because of the above-mentioned trade-off. However, it appears that central banks do not consider this potential conflict and its impact on capital allocation. During long-lasting recessions, ignoring this conflict may delay economic recovery to a certain extent and may derogate future growth possibilities because productive firms cannot actualize their full potential.

Our paper relates to the literature that addresses the fact that we observe neither an economic recovery nor extraordinary inflation despite low interest rates. Some authors argue that this is because we are in a liquidity trap (e.g., Schmitt-Grohé and Uribe (2012); Werning (2011); Mertens and Ravn (2011)).4 Others require certain additional

\footnotetext{
${ }^{2}$ Note that Schmitt-Grohé and Uribe (2012) also postulate a policy of increasing interest rates in their model with a liquidity trap to escape a jobless recovery.

${ }^{3}$ There is an ongoing discussion in the literature on whether central banks should also consider other goals, such as fighting asset price bubbles or stabilizing the financial system; see, e.g., Bernanke and Gertler (2001).

${ }^{4}$ There are similar papers for the case of Japan. See, e.g., Krugman (1998). 
assumptions, such as credit constraints (e.g., Guerrieri and Lorenzoni (2011) or Hall (2011)). Whereas these papers continue to use a short-run perspective, we provide an explanation for the phenomenon of an economy that no longer reacts to monetary stimuli from a medium-run perspective (i.e., flexible prices but sluggish capital stock or input shares used), as postulated by Solow (2000) or Blanchard (1997).

The rest of the paper is organized as follows. Section 2 provides an overview of the existing literature on the conventionally proposed medium- and long-run impacts of monetary policy. In section 3 , the components of the new model proposed in this study are developed, and section 4 describes how the long-run steady state is determined. Section 5 incorporates the central bank as a policy maker. For demonstrative purposes, a simulation is performed in section 6 to show the consequences of different monetary policies. Section 7 concludes.

\section{Review of literature}

Monetary policy generally finds its place only in research on business cycles in which New Keynesian models have been the primary workhorse for many years. This is a logical consequence of the generally accepted neutrality of monetary policy in the longrun economy. Authors who address the medium- or long-run impacts of monetary policy typically propose an indirect analysis by focusing on the potential consequences of the stabilizing effect of monetary policy.

Given the stabilizing effect of monetary policy, the relevant question now concerns the general relationship between business cycles and growth. The intellectual father of this idea is Joseph Schumpeter (1939), who formulated this concept as the "theory of creative destruction" in the 1930s. He stipulated that recessions are necessary to establish new technologies and production processes that will increase the long-run output of the economy. Whereas Schumpeter (1939) provided only a qualitative description, Aghion and Howitt (1992) brought the idea into a tractable model. The driving force of this model is the prospect of potential monopoly profits from new innovations that crowd out old inventions (representing the literal process of "creative destruction"). A similar mechanism is used in an earlier work by Aghion and Saint-Paul (1991).

Other authors have supported this view on the positive effects of recessions and therefore provide, at least implicitly, a critical view of the stabilizing policies of monetary authorities. Notably, the theoretical motivations differ substantially. Caballero and Hammour (1994) argue that creation or innovation is a costly process that is optimally smoothed over time. On the contrary, destruction is a cost-free process. Consequently, recessions (simply modeled as exogenous demand shocks) have a cleansing effect on the economy. Outdated units are destroyed, and there is simultaneously a relatively high rate of innovation. Essential to the outcome of the predictions by this model is the cost function of creation, which is treated as an exogenously given black box. Mortensen and 
Pissarides (1994) find similar results in the context of a search model that focuses on unemployment.

Conversely, there is also a wide body of literature that postulates a negative relationship between economic fluctuations and long-term growth. The theoretical reasoning for this branch of the literature resides in the effects of so-called "learning by doing", which was first proposed by Arrow (1962). A more recent model based on this idea was developed by Martin and Rogers (1997). Its rationale may be described as the positive external effect of production on future productivity. Given that this effect decreases with increasing production, there is a negative relationship between business-cycle volatility and productivity in the economy. Blackburn (1999) represents an essential contribution to the literature because he incorporated the "learning-by-doing" effect in a model with "creative destruction"; he merges the two models, which results in the finding that stabilization policy has a negative impact on growth capability.

Empirical results reveal an unclear picture and do not support either view. Gali and Hammour (1993), Saint-Paul (1993), and, more recently, Broda and Weinstein (2007) favor Schumpeter's hypothesis. The contrary position is mainly represented by Ramey and Ramey (1995), who found clear evidence of a negative relationship between volatility and growth in a sample of more than 95 countries. Martin and Rogers (2000) found a similar result when focusing on the cyclicality of unemployment. Although the empirical results are not conclusive, there is a tendency for micro-data, in particular, to support the "creative destruction" view (also postulated by Caballero and Hammour (1994)).

Due to developments following the financial crisis, more recent work focuses on the potential stabilizing impact of monetary policy and on the consequences of low interest rate policies and/or unconventional monetary policy instruments. ${ }^{5}$ Aghion, Farhi, and Kharroubi (2012) provide an empirical investigation that demonstrates that pro-cyclical real interest rates in interaction with credit constraints have a positive effect on labor productivity. Chu and Cozzi (2013) introduce a cash-in-advance constraint in a Schumpeterian $R \& D$ model. Due to the possibility of encouraging overinvestment in $R \& D$, a policy of low interest rates may be welfare decreasing. Contrary to the previous literature, this paper considers the medium-term effects of low interest rates by focusing on the survival rate of firms. Nevertheless, the proposed impact of monetary policy is related to the "creative destruction" hypothesis in a broader sense.

\section{The model}

The model presented here draws on Melitz (2003) and incorporates heterogeneous firms in the same style. ${ }^{6}$ The model is assumed to describe medium-run development in

\footnotetext{
${ }^{5}$ Some earlier studies did this implicitly by analyzing the welfare consequences of the Friedman rule. See Bhattacharya, Haslag, and Russell (2005) for an overview and a recent example.

${ }^{6}$ Bilbiie, Ghironi, and Melitz (2007) also attempted to analyze the effects of monetary policy with a Melitz-style model; however, they focused on the effect of fixed entry-costs in a standard DSGE 
an economy as proposed by Solow (2000) or Blanchard (1997). As related to the model, a fixed capital stock is assumed that cannot be decreased or increased in reaction to changing real interest rates that are determined by the central bank. ${ }^{7}$ Houses or machines cannot simply be liquidized or enhanced in the short-run economy, as is frequently modeled in macroeconomic research. In fact, these are time-consuming processes.

The model consists of a two-sector economy with monopolistic competition. Unanticipated shocks are the source of economic fluctuations and therefore are the justification for an active monetary policy. The model is static.

\subsection{Households}

It is assumed that the economy consists of a constant population of identical households normalized to one. These households own the total capital stock (denoted by $\bar{K}$ ) and all technologies in the economy. The preferences of a representative household are given by a Stone-Geary-style utility function over a continuum of goods indexed by $\omega{ }^{8}$

$$
U=\int_{\omega \in \Omega}[\ln (c(\omega)+q)-\ln (q)] d \omega
$$

where $c(\omega)$ describes consumption of a specific variety, and $q$ is a common preference parameter. The budget constraint reads as $\int_{\omega \in \Omega} p(\omega) c(\omega) d \omega \leq I$, where $I$ denotes the income of a household. It is assumed that $M$ varieties are produced in the economy. Households maximize their utility, which results in the following demand function for a specific variety:

$$
c(\omega)=p(\omega)^{-1}\left[\frac{I}{M}+q \bar{p}\right]-q,
$$

where $\bar{p} \equiv \frac{1}{M} \int_{\omega \in \Omega} p(\omega) d \omega$ is the average price of the consumed goods. Demand increases with average price, which can be interpreted as the relative price of the other goods, and higher income, and it decreases with higher price and greater product variety. These are standard properties in models with differentiated goods.

\footnotetext{
framework.

${ }^{7}$ This is generally a well-established approach and is used in older monetary models, in particular. See, for example, Barro and Gordon (1983) or Rogoff (1985).

${ }^{8}$ The Stone-Geary-style utility function is chosen because it allows for a more generalized form (i.e., variable elasticity of substitution) than the standard constant elasticity of substitution (CES) utility function, which is used by Melitz (2003), for example. Kongsamut, Rebelo, and Xie (2001) describe it as a short way to "embed different income elasticities in a parsimonious way" (p. 875). They also claim that this type of utility specification is supported empirically. Note that for $\lim _{q \rightarrow 0} U$, the utility function becomes CES with elasticity of substitution equals one.
} 


\subsection{Production}

Each firm produces a different variety, $\omega$. Capital must be borrowed from households at a common (net) interest rate, $r$. Production occurs by a linear constant-return-to-scale production function. All firms face identical fixed costs, $f>0$, but different marginal costs or, more precisely, different productivity levels that are denoted by $\varphi>0$. Firms draw their initial productivity $\varphi$ from a common continuous probability distribution $g(\varphi)$, which is defined over the interval $[0, \infty)$. The continuous cumulative distribution is $G(\varphi)$. Individual production is given by $y(\varphi)=\varphi(k(\varphi)-f)$. Thus, the pricing rule for every firm is the following:

$$
p(\varphi)=\sqrt{\frac{r}{\varphi q}\left[\frac{I}{M}+q \bar{p}\right]},
$$

based on its profit maximization considerations and depending on its individual productivity, $\varphi$. Given this pricing rule, the profit function of an individual firm reads as

$$
\pi(\varphi)=\left(\sqrt{\frac{I}{M}+q \bar{p}}-\sqrt{\frac{r q}{\varphi}}\right)^{2}-r f .
$$

This can be interpreted as the profit from regular production minus payment for fixed costs. Note that payment for fixed costs depends on the interest rate or, more technically, on the common marginal cost factor, which is how the interest rate can be interpreted. This is because fixed costs are paid in units of capital whose price varies with the interest rate.

\subsection{Zero cut-off profit condition}

To make profits, a firm must reach a minimum productivity level, $\varphi^{*}$ (cut-off productivity). Otherwise, the firm would drop out of the market. The price that a firm facing this cut-off productivity level charges is normalized to one $\left(p\left(\varphi^{*}\right)=1\right)$, i.e., this firm produces the numéraire. The cut-off productivity level is calculated by setting the profit function equal to zero and solving for $\varphi$ :

$$
\varphi^{*}=\frac{r}{1-\frac{r f}{\frac{I}{M}+q(\bar{p}-1)}}=\frac{r c\left(\varphi^{*}\right)}{c\left(\varphi^{*}\right)-r f} .
$$

It follows that $\frac{\partial \varphi^{*}}{\partial r}>0$ for every $r>0 .{ }^{9}$ This result is economically intuitive because it simply posits that the cut-off firm must reach a higher level of productivity with increasing costs of borrowing capital to stay in the market. The most important consequence of this process is that some capital resources become available after an increase of

\footnotetext{
${ }^{9}$ Formal proof is part of Appendix B.1.
} 
$r$ that can be reallocated to other firms that are more productive. Furthermore, for every producer that drops out of the market, unproductive fixed costs can be avoided. Unfortunately, one would also have to accept a reduced number of varieties because production is more expensive.

\subsection{Aggregation}

As previously discussed, equilibrium is characterized by a mass $M$ of firms (and therefore varieties). This mass is based on a finite total number of potential goods $\omega \in \Omega$, which is defined as $M_{0}$. It is self-evident to assume the existing technologies - and therefore the potential varieties - as given in a static medium-run model because developing new technologies is a time-consuming and dynamic process. ${ }^{10}$ Because a fraction $G\left(\varphi^{*}\right)$ of all firms does not produce, the mass of varieties is given by

$$
M=M_{0}\left(1-G\left(\varphi^{*}\right)\right) .
$$

Given the share of non-producing firms, $G\left(\varphi^{*}\right)$, the distribution of producing firms is conditional on $\left[\varphi^{*}, \infty\right)$. The average price $\bar{p}$ is given by

$$
\bar{p}=\frac{1}{M} \int_{\varphi^{*}}^{\infty} p(\varphi) M \frac{g(\varphi)}{1-G\left(\varphi^{*}\right)} d \varphi .
$$

Inserting the pricing equation (3), we implicitly obtain the productivity of a firm charging $\bar{p}$ :

$$
\tilde{\varphi}\left(\varphi^{*}\right):=\left(\frac{1}{1-G\left(\varphi^{*}\right)} \int_{\varphi^{*}}^{\infty} \frac{1}{\sqrt{\varphi}} g(\varphi) d \varphi\right)^{-2} .
$$

Note that $\tilde{\varphi}$ is an important reference for the productivity of the economy. However, contrary to Melitz (2003), there is no possible direct link to total production in the economy. This is because $\tilde{\varphi}$ is a pure average productivity measure (not a weighted measure).

Solving the quadratic equation (7) for $\bar{p}$ gives us the average price charged in this economy:

$$
\bar{p}=\frac{1}{2} \sqrt{\frac{r}{\tilde{\varphi}}}\left(\sqrt{\frac{r}{\tilde{\varphi}}} \pm \sqrt{\frac{r}{\tilde{\varphi}}+\frac{4 I}{M q}}\right)
$$

Since prices cannot be negative, only the positive solution of this equation is feasible. The average price level rises with a higher interest rate indicating the shifting of higher production costs to the Because prices cannot be negative, this equation may only have a positive solution. The average price level rises with a higher interest rate, which indicates

${ }^{10}$ Note that Dixit and Stiglitz (1977) implicitly use the same approach. 
that production costs are shifted to consumers. Furthermore, $\bar{p}$ increases as household income rises but decreases with a higher number of producing firms, i.e., with stronger competition. In what follows, this average price is treated as a measure for the general price level; that is, it works as a proxy for a price index. ${ }^{11}$

Because the entire production is consumed, we can sum up over the continuum of all producing firms by using the demand function (2) and the pricing rule (3), obtaining total output $Y$ :

$$
Y=M\left(\sqrt{\frac{q}{r}\left[\frac{I}{M}+q \bar{p}\right]} \int_{\varphi^{*}}^{\infty} \sqrt{\varphi} \frac{g(\varphi)}{1-G\left(\varphi^{*}\right)} d \varphi-q\right) .
$$

Note that $\frac{\partial Y}{\partial r}>0 \forall r>0$, but $\frac{\partial Y}{\partial f}<0$. The first result is not surprising because it is a central feature of the model that a higher interest rate encourages the reallocation of capital from less to more productive firms. ${ }^{12}$ However, the latter result is not as obvious. It should be borne in mind that higher fixed costs always affect the entire economy, not only relatively unproductive firms (which are, however, most likely to exit after a rise in fixed costs). That is, potential newly available capital is more than completely depleted by higher fixed costs.

The last missing piece to close the model is household income. Households receive an interest rate payment for lending their capital and, because they are their owners, all the profits that firms make. Note that lending to firms is the only way that households can use their capital; the entire capital stock $\bar{K}$ is always used in production. Utilizing the individual profit function (4), this means

$$
I=r \bar{K}+\int_{\varphi^{*}}^{\infty} M \pi(\varphi) \frac{g(\varphi)}{1-G\left(\varphi^{*}\right)} d \varphi=r \bar{K}+M\left(\frac{I}{M}-\bar{p} q+r q \bar{\varphi}-r f\right)
$$

where $\bar{\varphi} \equiv \int_{\varphi^{*}}^{\infty} \frac{1}{\varphi} \frac{g(\varphi)}{\left(1-G\left(\varphi^{*}\right)\right)} d \varphi$.

Using the definition (9) for $\bar{p}$, we obtain

$$
I=r\left(\frac{(\bar{K}+M(q \bar{\varphi}-f))^{2} \tilde{\varphi}-(\bar{K}+M(q \bar{\varphi}-f)) M q}{M q^{2}}\right) .
$$

Equation (12) implies a direct one-to-one effect of the interest rate, $r$, on household income as a consequence of the income of capital lending. However, a change in $r$ also affects the cut-off productivity $\varphi^{*}$. This has two effects. On the one hand, the measure for the average productivity $\tilde{\varphi}$ becomes higher, which leads to an increase in production and profits per firm; on the other hand, the number of firms decreases, and fewer firms can pay their profits to households. The first effect dominates. This is economically

\footnotetext{
${ }^{11}$ The same strategy is used by Melitz and Ottaviano (2008).
}

${ }^{12} \mathrm{~A}$ formal proof is included as part of Appendix B.3. 
intuitive because total production is strictly increasing in $r$, i.e., the potential profit base increases. ${ }^{13}$

Because the definition for household income closes the model, the following proposition can be made.

Proposition 1. For any given set of parameters $\left[r>0, f>0, q>0, \bar{K}>0, M_{0}>0\right]$ and distribution $G(\varphi)$ defined over the interval $[0, \infty)$, the economy is characterized by a unique and stationary equilibrium.

Proof. See Appendix B.1.

Contrary to Melitz (2003), in equilibrium, the firm level determinant $\varphi^{*}$ is not independent of the size of the economy, which can be approximated by $\bar{K}$. A higher capital stock enables more firms to stay in the market. This is a consequence of different preferences. For the interpretation of the equilibrium at this point, consider the interest rate $r$ and the capital stock $\bar{K}$ as exogenously given. Section 4 provides an illustration of their formation.

\subsection{Welfare analysis}

Welfare is determined by two factors in this economy, as it is in most models with monopolistic competition. First, individual utility increases with the number of consumed units of each product, whereas the marginal return of each additional unit consumed is diminishing. Second, consumers are variety lovers; they enjoy a wider range of differentiated products from which they can select. Given a specific equilibrium, welfare is given by the utility of the representative household,

$$
U=\frac{M_{0}}{2}\left[\int_{\varphi^{*}}^{\infty} \ln (\varphi) g(\varphi) d \varphi+\left(1-G\left(\varphi^{*}\right)\right) \ln \left(\frac{I}{r q M}+\frac{\bar{p}}{r}\right)\right] .
$$

An increase in $r$ leads to an amplification of production as well as a reduction in the number of producing firms. Welfare, however, depends on both dimensions, which leads to the following proposition.

Proposition 2. $\exists$ a welfare-maximizing interest rate $\mathfrak{r} \in[0, \infty)$.

\section{Proof. See Appendix B.2.}

This indicates that there is an interest rate that ensures the optimal combination of production volume and variety. This result is consistent with the general trade-off of

${ }^{13}$ Formal proof is included as part of Appendix B.1. 
quantity versus diversity in monopolistic competition models, which was first described by Dixit and Stiglitz (1977), who also propose that there is an optimal allocation of existing resources. As opposed to their approach of influencing the allocation by constraining the number of firms, this effect can result from adjusting the interest rate in our model.

\section{The long-run steady state}

Thus far, the interest rate $r$ and the capital stock $\bar{K}$ have been treated as free parameters without economic substantiation. This is now changed, and we now put the model in a dynamic Ramsey-Cass-Koopmans $[\mathrm{RCK}]^{14}$ framework, which enables us to investigate how capital stock is accumulated and how the long-run interest rate is determined. Individuals face the following long-run dynamic optimality condition:

$$
\max _{C(t), K(t)} \int_{0}^{\infty}[e^{-\rho t} U(C(t))+\mu(t) \underbrace{(I(t)-C(t))}_{=\dot{K}(t)}] d t
$$

where $t$ is the time index, $\rho$ is the time preference of individuals, $\mu(t)$ is the Lagrange multiplier, and $U(C(t))$ is the utility from total consumption in one period ${ }^{15}$. Because we are only interested in the steady state, we focus on the corresponding asset-pricing equation. Individuals do not take into account the impact of their capital accumulation on the profits generated by firms but only on the return of capital, $r .{ }^{16}$ This yields the following optimization rule:

$$
\dot{\mu}(t)=\rho \mu(t)-\frac{\partial[r(t) K(t)]}{\partial K(t)} \mu(t) .
$$

In a steady state, growth rates are zero, i.e., $\dot{\mu}(t)=0 .{ }^{17}$ Solving for $r$ yields the steady state interest rate, denoted as $r^{*}$ (the time index is dropped in what follows because we are in a steady state): $r^{*}=\rho$, which is the standard result in RCK models and simply indicates that individuals save until the return of additional savings equals their time preference rate.

The (fixed) steady-state capital stock can finally be determined from the fact that the entire production must be consumed in the steady state (note that this is also the case in the baseline model from section 3). From the production function, we know that

\footnotetext{
${ }^{14}$ c.p. Ramsey (1928), Cass (1965), Koopmans (1965)

${ }^{15}$ i.e., $U(C(t))=\int_{\omega \in \Omega}[\ln (c(\omega, t)+q)-\ln (q)] d \omega$.

${ }^{16}$ The "social planner", on the contrary, would do exactly that. His optimization problem and solution is presented in Appendix C.

${ }^{17}$ It is assumed that the usual transversality conditions apply.
} 
the capital used by a single firm is given by $k(\varphi)=\frac{c(\varphi)}{\varphi}+f$. Summing up over all firms gives us the capital stock for the entire economy,

$$
\bar{K}=\int_{\varphi^{*}}^{\infty} M k(\varphi) \frac{g(\varphi)}{1-G\left(\varphi^{*}\right)} d \varphi=M\left(\sqrt{\frac{q}{\rho \tilde{\varphi}}\left[\frac{I}{M}+q \bar{p}\right]}-q \bar{\varphi}+f\right) .
$$

This closes the steady state version of the model (all other equations from section 3 continue to apply, i.e., all other variables are defined by the respective definitions) and determines the values for $r^{*}$ and $\bar{K}$ for a given set of parameters. It is important to note that the long-run interest rate, $r^{*}$, does not equal the welfare-maximizing interest rate, r.

\section{The central bank}

This section examines the potential impact of monetary policy on our stylized economy. It is assumed that monetary policy is implemented by a non-discretionary central bank, which uses the interest rate as its objective function. As is standard, the central bank aims for price and output stability. ${ }^{18}$

To capture our basic idea, we assume that the central bank is able to distort the real interest rate. We model this concept as follows: the central bank enforces its policy by imposing a tax (subsidy) $\tau$ on the interest rate paid by firms. ${ }^{19}$ Tax revenues (subsidy expenses) are redistributed to (taken from) households. Therefore, interest payment for firms is now given by

$$
r k(\varphi)=\hat{r}(1+\tau) k(\varphi)
$$

where $\hat{r}$ is the market interest rate. Given the capital stock $\bar{K}$, the tax generates a revenue of $\tau \hat{r} \bar{K} \equiv T$ (in case of a subsidy, the amount must be taken from the households in the form of a lump sum tax). ${ }^{20}$ Household income therefore becomes

$$
I=\hat{r} \bar{K}+\int_{\varphi^{*}}^{\infty} M \pi(\varphi) \frac{g(\varphi)}{1-G\left(\varphi^{*}\right)} d \varphi+T=r \bar{K}+\int_{\varphi^{*}}^{\infty} M \pi(\varphi) \frac{g(\varphi)}{1-G\left(\varphi^{*}\right)} d \varphi .
$$

This equals the definition for household income from (11), which implies that the properties from our model above, in which no explicitly defined interest rate has been

\footnotetext{
${ }^{18}$ Note that price stability in a narrow sense does not affect welfare. However, central bank policy (which includes the central bank's reaction to changes in the price level) does have an impact on welfare.

${ }^{19}$ Imposing a tax is normally a fiscal act. However, we consider the process a monetary projection for two reasons. First, it is assumed that the policy follows a strict rule, as is normally assumed only for central banks. Second, monetary policy is sometimes assumed to have fiscal effects, such as in Darby (1975).

${ }^{20}$ Note that this only holds for the medium-run economy with a constant capital stock. In the long-run economy, the capital stock would adjust when a tax is imposed on the interest rate, as in the standard RCK model. See, e.g., Barro (1990) for a discussion.
} 
used, hold. By setting the tax according to the rule $\tau=\frac{r-\hat{r}}{\hat{r}}$, the central bank is able to implement every feasible interest rate $r$.

Proposition 3. By imposing tax rate $\tau \in[-1, \infty)$ on the interest rate paid by firms whose revenues (or expenses) are redistributed to (or taken from) households, the central bank can implement any interest rate, $r$, that it desires.

Proof. Proven in text.

Thus, in what follows, we treat the interest rate as freely settable by the central bank and relinquish an explicit formulation of the tax rate. This approach improves readability without loss of generality.

The central bank is supposed to react to real, non-permanent shocks on the economy. These might be preference shocks, cost shocks, or supply-side shocks to the productivity distribution. Monetary policy reacts to these disturbances by strictly following a simple Taylor-style rule, given by

$$
r=r^{*}\left(\frac{\bar{p}}{\bar{p}^{*}}\right)^{\psi}\left(\frac{Y}{Y^{*}}\right)^{\gamma} .
$$

$\bar{p}^{*}$ and $Y^{*}$ denote the average price and the output level in the absence of any shocks (or in the long-run steady state), respectively. $\psi$ and $\gamma$ are weighting parameters that describe the reaction of monetary policy to deviations from the long-run steady state. In a standard monetary framework, the signs of $\psi$ and $\gamma$ would be positive, i.e., the central bank would raise interest rates when the price levels and output are above the steadystate levels and would decrease them if they are below the steady-state levels after the shock.

In this model, however, such a reaction would accelerate the deviation because output and price level are positively correlated with the interest rate. The first observation has been previously explained and is caused by a higher interest rate leading to a reallocation of capital to more productive firms. The second observation is caused by the interest rate working as a common marginal cost factor. An increase would be shifted to consumer prices. A new configuration of monetary policy design is therefore necessary.

Proposition 4. A stabilizing monetary policy requires $\psi, \gamma<0$, which implies an inverse Taylor rule for monetary policy.

Proof. See Appendix B.3.

This observation implies a trade-off in the stabilization goal of the central bank between the short-run and the medium-run economies. After a negative demand shock, for example, low interest rates might work to stabilize in the short-run economy. However, 
in a case with a long-lasting shock, lax monetary policy will most likely delay the reallocation of capital in the medium-run economy by tiering resources at unproductive firms. This lowers production capacity within the economy. Moreover, the monetary authority would possibly prolong economic downturn relative to non-intervention by maintaining a low interest rate.

It is important to note that the welfare aspect of a stabilizing monetary policy is ambiguous. Given that $r^{*}>\mathfrak{r}$ (i.e., the steady state interest lies above the welfaremaximizing interest), stabilization after a negative shock might not be a desirable policy because of the preference for diversity. This depends on how welfare reacts to a specific shock. Whereas output volume is always affected in the direction of the shock, the impact on diversity (which also affects welfare) is arbitrary. This directly implies that stabilization might be welfare improving after a positive shock because it can increase diversity, although stabilization itself has no intrinsic value for the individuals in the model. The following section provides some insights.

\section{Numerical simulations}

The impact of monetary policy on welfare differs depending on the policy parameters $\psi$ and $\gamma$ in addition to depending on the settlement of the steady state interest rate, $r^{*}$, relative to the welfare-maximizing interest rate, $\mathfrak{r}$, after a potential shock. To qualitatively demonstrate these effects, some simulations are performed that show the consequences for welfare under specific monetary policy rules (without any pretensions of a quantitative predication). The model is not analytically solvable due to fundamental non-linearities, i.e., a numerical algorithm is implemented. ${ }^{21}$

It is assumed that productivities are exponentially distributed, i.e., $\varphi \sim \exp (\lambda)$. The exponential distribution is used for two reasons. First, from an economical point of view, $g(\varphi)$ is strictly decreasing in $\varphi$, which represents a comprehensible view of reality because relatively productive ideas are rarer then relatively unproductive ideas. Second, this type of distribution is supported over the entire theoretical interval $[0, \infty)$, i.e., it fits the presumptions of the model. This is a clear advantage relative to the Pareto distribution, which is sometimes used in similar setups. ${ }^{22}$

The chosen parameters allow a differentiation between the two cases discussed above $\left(r^{*}<\mathfrak{r}\right.$ and $\left.r *>\mathfrak{r}\right)$. Nevertheless, in both simulations, a demand-side preference shock to $q$ is examined. Noting $q^{*}$ as the steady state value of $q$, it follows that $q=q^{*} \varepsilon$, where $\varepsilon$ is a positive idiosyncratic shock with a mean of one. The central bank reacts to the shock by setting the interest rate according to the Taylor rule. To evaluate the effects of monetary policy, the Taylor rule parameters, $\psi$ and $\gamma$, are alternated in the

\footnotetext{
${ }^{21}$ The algorithm is based on a numerical convergence. The respective MatLab-Code is available upon request.

${ }^{22}$ e.g., Melitz and Ottaviano (2008).
} 
simulation. Because we know from proposition 4 that the stabilization of output and price level requires $\psi, \gamma<0$, the parameters are allowed to be positive. However, we make the restriction that $|\psi|<1$ and $|\gamma|<1$, i.e., the marginal reaction of the central bank will not be increasing.

\subsection{Monetary policy with $r^{*}<\mathfrak{r}$}

We begin with the case in which the steady state interest rate lies below the welfaremaximizing level in the steady state. Regarding parameters, we set $\lambda$ to 0.5 , which allows for a relatively smooth distribution of productivities. The number of potential ideas, $M_{0}$, is normalized to one. The preference parameter, $q$, is also set to one. Fixed costs $f$ are set to 20, which simply represents an upscaling for illustrational purposes. The time preference parameter, $\rho$, is assumed to be 0.04 , which implies a realistic long-run interest rate, $r^{*}$ of $4 \%$. This combination of parameters implies a steady-state capital stock, $\bar{K}$ of 36.68. The welfare-maximizing interest rate $\mathfrak{r}$ in this case is 0.32 (i.e., $32 \%$ ), which is far higher than $r^{*}$ and thus implies that the case with $r^{*}<\mathfrak{r}$ is the more plausible one. Now, we assume that this model economy is hit by a shock $\varepsilon=1.05$.

As the model suggests - and consistent with the data - such a demand shock leads to a decline in output and the average price level. The same holds for the number of firms producing and for the utility level, although the effect on the former is very small. ${ }^{23}$ Figure 2 shows the welfare level relative to the steady state (as a percentage) in dependence on the parameters $\psi$ and $\gamma$.

${ }^{23}$ However, this is primarily a consequence of the parameters chosen. 


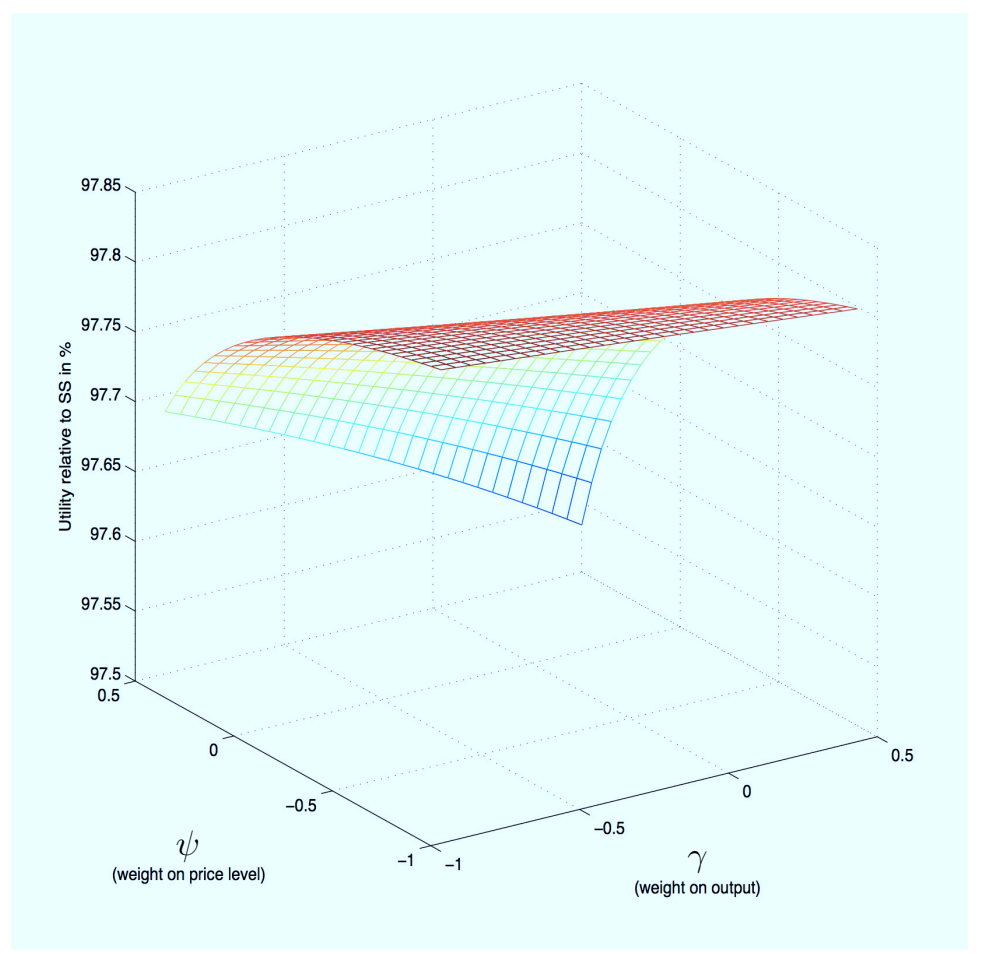

Figure 2: Utility after a preference shock depending on monetary policy with $r^{*}<\mathfrak{r}$.

The figure shows that welfare constantly increases with lower policy parameters; that is, the stabilizing, inverse Taylor rule is also welfare improving after a negative shock. This is the case as long as the policy parameters ensure that $r(\psi, \gamma) \leqslant \mathfrak{r}$. This result represents production increasing as a result of the reallocation of capital to relatively more productive firms initialized by the central bank. Note that the effect is non-linear; the marginal impact of a decrease in the policy parameters is diminishing. Individuals rank higher consumption possibilities higher than the loss of variety in this example. As a consequence, there is no conflict between the stabilization goal of the central bank and welfare.

\subsection{Monetary policy with $r^{*}>\mathfrak{r}$}

To understand the model's mechanism, we also investigate the case in which the longrun interest rate is higher than the welfare-maximizing rate. In particular, we increase the time-preference rate $\rho$ to 0.4. It is clear that this implies a very high and rather unrealistic discount rate. All other parameters remain the same, yielding a steady state capital stock $\bar{K}$ of 34.71 and a welfare-maximizing interest rate $\mathfrak{r}$ of 0.35 . Again, we assume that the model economy is hit by a demand shock $\varepsilon=1.05$. Figure 3 shows the 
welfare level relative to steady state (as a percentage) in dependence on the parameters, $\psi$ and $\gamma$, for this different situation.

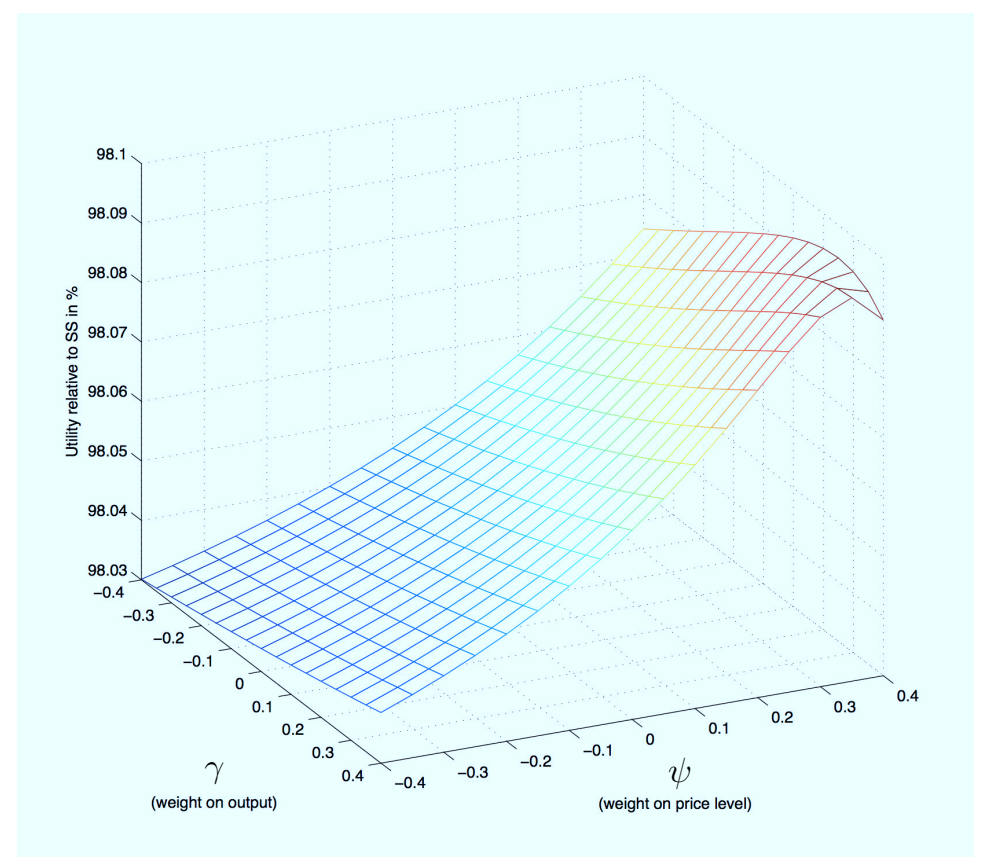

Figure 3: Utility after a preference shock depending on monetary policy with $r^{*}>\mathfrak{r}$.

Contrary to the case described above, welfare-optimizing policy now requires positive values for $\psi$ and $\gamma$, although this would shift the economy further away from its steady state. The reason for this is the preference for variety of individuals. From their perspective, monetary policy should permit more firms to enter the market regardless of their relatively low productivity. This example reveals an important insight: stabilizing monetary policy is not always welfare improving in the model. The impact on utility depends on the position of the steady-state interest rate relative to the welfare-maximizing interest rate.

Despite this theoretically interesting thought experiment, which must be interpreted in light of the very high time preference rate, we must keep in mind that monetary policy cannot directly influence the structure of the economy in the long-run. This implies that a monetary policy that further amplifies the variance from steady state can never be sustainable. A central bank that wants to stabilize output and the price level in the model should therefore follow the inverse Taylor rule (discussed above) in any case. 


\section{Conclusion}

In light of the ongoing and long-lasting period of low interest rates worldwide, we have developed a monopolistic competition model with heterogeneous firms to investigate the impact of monetary policy in the medium-run economy. "Medium run" has been interpreted in the sense that prices are flexible, but the capital stock and the potential amount of variety are assumed to be fixed because adjustments are time consuming. The driving factor is the interest rate through its role as the common marginal cost factor. An increase in the interest rate (induced by the central bank) forces relatively unproductive firms to leave the market because they would make negative profits. This allows for capital to be reallocated to relatively more productive firms, which would lead to higher productivity on average and, therefore, to higher output in the economy.

Because of this channel, the model suggests that an output-stabilizing monetary policy should increase interest rates such that the reallocation of capital within the economy in a long-lasting crisis is not delayed. Furthermore, the price level reacts positively to a rising interest rate in the model because of its nature as a common marginal cost factor. This phenomenon may have important implications for monetary authorities because their policy goal is stabilization. Their medium-run Taylor rule becomes inverse, which indicates that they should slow down expansionary monetary policy at some point in a long-lasting crisis. In other words, central banks might face a trade-off in their stabilization goal between the short-run and medium-run economies. Note that this insight does not imply that monetary authorities should simply induce a sharp switchover of their policy after some time because this would most likely lead to negative short-run distortions. Instead, it suggests a smooth revaluation of monetary policy instruments.

It should be noted that excessive promotion of the reallocation of capital to very productive firms would not be optimal from a welfare perspective. In the context of the model, this is a consequence of the preference for variety. Conversely, the model theoretically suggests that it might be welfare improving, under certain circumstances, to further amplify the deviation from the steady-state economy to increase diversity. However, such policies would never be sustainable because the impact of monetary policy diminishes in the long-run economy.

Further research must be conducted regarding the impact of monetary policy on the structural patterns of an economy. On the one hand, future theoretical work might analyze the mechanism in a more dynamic environment. In particular, the introduction of heterogeneous firms - as presented here - in a classical New Keynesian framework seems promising. The basic premise that the manipulation of the interest rate by the central bank has an impact on whether a specific firm can stay in the market can be maintained. On the other hand, empirical research might investigate the impact of monetary policy on the structure in particular industries. How do firm entry and exit rates react to a specific interest rate policy when controlling for other factors, and what is the impact on overall productivity? With firm-level data, such an investigation should be possible. 


\section{Appendix A. Structural breaks in U.S. real interest rates.}

The test for a structural break in the real interest rates in the United States is based on a regression of the following form

$$
T I P S_{i, t}=\beta_{i}^{1}+\beta_{i}^{2} T I P S_{i, t-1}+\beta_{i}^{3} R_{t}+\varepsilon_{i, t},
$$

where $t$ is a time index, $i$ denotes the maturity, and $R$ is the federal fund rate. ${ }^{24}$ It is assumed that a structural break in the constant $\beta_{i}^{1}$ indicates a change in the long-term real interest rate. A Quandt-Andrews unknown breakpoint test with $10 \%$ trimming is performed for two samples: one for before the crisis (January 2003-January 2007) and one for the entire available sample (January 2003-January 2013). Table A.1 summarizes the results.

Table A.1: Test for a structural break in the real interest rate.

\begin{tabular}{l|c|c}
\hline \hline & \multicolumn{2}{|c}{ Sample } \\
\hline Maturity & $2003 \mathrm{M} 01-2007 \mathrm{M} 01$ & $2003 \mathrm{M} 01-2013 \mathrm{M} 01$ \\
\hline 5 years & 8.78 & $13.17^{* *}$ \\
& $(2004 \mathrm{M} 08)$ & $(2008 \mathrm{M} 12)$ \\
\hline 7 years & $9.36^{*}$ & $13.55^{* *}$ \\
& $(2004 \mathrm{M} 08)$ & $(2008 \mathrm{M} 12)$ \\
\hline 10 years & 8.57 & $12.6^{* *}$ \\
& $(2004 \mathrm{M} 08)$ & $(2011 \mathrm{M} 03)$ \\
\hline 20 years & 5.93 & $15.07^{* *}$ \\
& $(2006 \mathrm{M} 03)$ & $(2011 \mathrm{M} 07)$ \\
\hline \hline
\end{tabular}

Notes: Test statistics correspond to the maximum LR F-statistics. $* *$ and $*$ indicate rejection of the null hypothesis of no break point at the $1 \%$ and $5 \%$ significance levels, respectively. Critical values correspond to Hansen (1997). Suggested break points are in parenthesis.

Test statistics mostly imply a structural break for the longer period but not for the period before the crisis (for the 7-year T-Bill; the test statistic is narrowly significant at the $5 \%$ significance level for the shorter period). This is an indicator of a monetary policy that is able to influence real interest rates over a long period. It is clear that other explanations, such as changing time preferences, may also play a role.

\footnotetext{
${ }^{24}$ Monthly data on real interest rates are TIPS data (i.e., inflation-protected treasury bills) from the U.S. Department of the Treasury. Data on the federal fund rate are from the FED.
} 


\section{Appendix B. Proofs.}

\section{Appendix B.1. Proof of Proposition 1.}

Proof. In the following, we show the existence and uniqueness of the equilibrium and the increase of $\varphi^{*}$ in $r$.

\section{Step 1: Definitions.}

For an easier notation, let us define

$$
\frac{I}{M}:=r \underbrace{\left(\frac{(\bar{K}+M(q \bar{\varphi}-f))^{2} \tilde{\varphi}-(\bar{K}+M(q \bar{\varphi}-f)) M q}{(M q)^{2}}\right)}_{\equiv B}=r B .
$$

This implies

$$
\bar{p}:=\frac{1}{2} \sqrt{\frac{r}{\tilde{\varphi}}}\left(\sqrt{\frac{r}{\tilde{\varphi}}}+\sqrt{\frac{r}{\tilde{\varphi}}+\frac{4 r B}{q}}\right)=r \underbrace{r \frac{1}{2}\left(\frac{1}{\tilde{\varphi}}+\sqrt{\left.\frac{1}{\tilde{\varphi}^{2}}+\frac{4 B}{q}\right)}\right.}_{\equiv D}=r D .
$$

The equilibrium of the economy can be described in a single equation by inserting equations (12) and (9) into the equation for the cut-off productivity (5). This yields the following definition:

$$
\varphi^{*}=\frac{r}{1-\frac{r f}{c\left(\varphi^{*}\right)}}=\frac{r}{1-\frac{r f}{r B+q(r D-1)}}=\frac{r B+r q D-q}{B+q D-\frac{q}{r}-f}:=\mathfrak{f}\left(r, \varphi^{*}\right) .
$$

Given that there is a single $\varphi^{*}$ for a given set of parameters $[r>0, f>0, q>0 \bar{K}>$ $\left.0, M_{0}>0\right]$ and distribution $g(\varphi)$ defined over the interval $[0, \infty)$, that solves this equation, and the economy is characterized by a unique and stationary equilibrium.

Step 2: Boundary values.

In this step, the boundary values for $\mathfrak{f}\left(r, \varphi^{*}\right)$ are calculated. Note that $B>0$ and, therefore, $D>0 \forall \varphi^{*} \in[0, \infty)$. Furthermore, it holds that $\lim _{\varphi^{*} \rightarrow \infty} B, D=\infty$, implying that

$$
\lim _{\varphi^{*} \rightarrow \infty} \mathfrak{f}\left(r, \varphi^{*}\right)=r
$$

which is the lowest possible value for $\varphi^{*}$ (the same result obtains in the absence of fixed costs. In this case, we would have $\left.\mathfrak{f}\left(r, \varphi^{*}\right)=r \forall \varphi^{*}\right)$. 
Moreover, we have $\lim _{c\left(\varphi^{*}\right) \rightarrow r f} \mathfrak{f}\left(r, \varphi^{*}\right)=\infty$, or, more precisely,

$$
\lim _{\varphi^{*} \rightarrow c^{-1}(r f)} \mathfrak{f}\left(r, \varphi^{*}\right)=\infty,
$$

which is the highest possible value for $\varphi^{*}$. This implies that $\mathfrak{f}\left(r, \varphi^{*}\right)$ is decreasing from $\infty$ to $r$ as $\varphi^{*}$ is increasing from $c^{-1}(r f)$ to $\infty$.

\section{Step 3: Derivatives.}

If we can show that $\frac{\partial \mathfrak{f}\left(r, \varphi^{*}\right)}{\partial \varphi^{*}} \leqslant 0 \forall \varphi^{*} \in\left[c^{-1}(r f), \infty\right)$, we have a unique equilibrium (because the left-hand side of (B.3) is monotonically increasing).

The derivative of $\mathfrak{f}\left(r, \varphi^{*}\right)$ is given by

$$
\frac{\partial \mathfrak{f}\left(r, \varphi^{*}\right)}{\partial \varphi^{*}}=\frac{-r f\left(r \frac{\partial B}{\partial \varphi^{*}}+r q \frac{\partial D}{\partial \varphi^{*}}\right)}{\left(c\left(\varphi^{*}\right)-r f\right)^{2}}=\frac{-f\left(\frac{\partial B}{\partial \varphi^{*}}+q \frac{\partial D}{\partial \varphi^{*}}\right)}{\left(B+q D-\frac{q}{r}-f\right)^{2}} .
$$

This expression is non-positive as long as $r \frac{\partial B}{\partial \varphi^{*}}+r q \frac{\partial D}{\partial \varphi^{*}} \geqslant 0$. To show this, let us first provide the derivatives of some variables:

$$
\begin{aligned}
\frac{\partial M}{\partial \varphi^{*}} & =-g\left(\varphi^{*}\right) M_{0}<0 \\
\frac{\partial \bar{\varphi}}{\partial \varphi^{*}} & =\frac{g\left(\varphi^{*}\right)}{1-G\left(\varphi^{*}\right)}\left[\bar{\varphi}-\frac{1}{\varphi^{*}}\right]<0 \\
\frac{\partial \tilde{\varphi}}{\partial \varphi^{*}} & =2 \tilde{\varphi}^{\frac{2}{3}} \frac{g\left(\varphi^{*}\right)}{1-G\left(\varphi^{*}\right)}\left[\frac{1}{\sqrt{\varphi^{*}}}-\tilde{\varphi}^{-\frac{1}{2}}\right]>0
\end{aligned}
$$

These are now used to calculate the derivative of $B$ :

$$
\begin{aligned}
\frac{\partial B}{\partial \varphi^{*}}= & \frac{1}{(M q)^{4}}[2(\bar{K}+M(q \bar{\varphi}-f)) \overbrace{\left(M_{0} g\left(\varphi^{*}\right)\left(f-\frac{q}{\varphi^{*}}\right)\right)}^{\frac{\partial M}{\partial \varphi^{*}} q \bar{\varphi}+M q \frac{\partial \bar{\varphi}}{\partial \varphi^{*}}-\frac{\partial M}{\partial \varphi^{*}} f} \\
& \frac{\partial \tilde{\varphi}}{\partial \varphi^{*}}(\bar{K}+M(q \bar{\varphi}-f))^{2}(M q)^{2}+g\left(\varphi^{*}\right) M_{0} q(\bar{K}+M(q \bar{\varphi}-f))(M q)^{2}- \\
& \left(M_{0} g\left(\varphi^{*}\right)\left(f-\frac{q}{\varphi^{*}}\right)\right)(M q)^{3}+\underbrace{2 M g\left(\varphi^{*}\right) M_{0} q^{2}}_{-\frac{\partial(M)^{2}}{\partial \varphi^{*}}}\left((\bar{K}+M(q \bar{\varphi}-f))^{2} \tilde{\varphi}\right. \\
& -(\bar{K}+M(q \bar{\varphi}-f)) M q)] .
\end{aligned}
$$

It holds that $\frac{\partial B}{\partial \varphi^{*}}>0$. This is a consequence of the fact that $\varphi^{*} \geqslant c^{-1}(r f)$. It implies $f-\frac{q}{\varphi^{*}} \geqslant 0$. Assume $f \geqslant \frac{q}{c^{-1}(r f)}$, then it must be that $c^{-1}(r f) \geqslant \frac{q}{f} \Leftrightarrow r f \geqslant c\left(\frac{q}{f}\right)$. This is can be observed by inserting $\varphi=\frac{q}{f}$ into the profit function (4). It becomes negative, 
implying that $r f \geqslant c\left(\frac{q}{f}\right)$.

Furthermore, the derivative of $D$ is given by

$$
\begin{aligned}
\frac{\partial D}{\partial \varphi^{*}} & =-\frac{1}{2 \tilde{\varphi}^{2}} \frac{\partial \tilde{\varphi}}{\partial \varphi^{*}}+\frac{1}{4 \sqrt{\frac{1}{\tilde{\varphi}^{2}}+\frac{4 B}{q}}}\left(-2 \frac{1}{\tilde{\varphi}^{3}} \frac{\partial \tilde{\varphi}}{\partial \varphi^{*}}+\frac{4}{q} \frac{\partial B}{\partial \varphi^{*}}\right) \\
& =-\frac{1}{2 \tilde{\varphi}^{3}} \frac{\partial \tilde{\varphi}}{\partial \varphi^{*}}\left(\tilde{\varphi}+\frac{1}{\sqrt{\frac{1}{\tilde{\varphi}^{2}}+\frac{4 B}{q}}}\right)+\frac{\frac{\partial B}{\partial \varphi^{*}}}{q \sqrt{\frac{1}{\tilde{\varphi}^{2}}+\frac{4 B}{q}}} \\
& =\frac{\frac{\partial B}{\partial \varphi^{*}} \frac{1}{q} 2 \tilde{\varphi}^{3}-\frac{\partial \tilde{\varphi}}{\partial \varphi^{*}}\left(1+\tilde{\varphi} \sqrt{\frac{1}{\tilde{\varphi}^{2}}+\frac{4 B}{q}}\right)}{2 \tilde{\varphi}^{3} \sqrt{\frac{1}{\tilde{\varphi}^{2}}+\frac{4 B}{q}}} .
\end{aligned}
$$

Using (B.10) (the second addend), it can be shown that $\frac{\partial D}{\partial \varphi^{*}}>0$. It is a consequence of the fact that

$$
\begin{gathered}
\frac{\partial \tilde{\varphi}}{\partial \varphi^{*}} \frac{2 \tilde{\varphi}^{3} \frac{1}{q}(\bar{K}+M(q \bar{\varphi}-f))^{2}}{(M q)^{2}}>\frac{\partial \tilde{\varphi}}{\partial \varphi^{*}}\left(1+\tilde{\varphi} \sqrt{\frac{1}{\tilde{\varphi}^{2}}+\frac{4 B}{q}}\right) \Leftrightarrow \\
\frac{\tilde{\varphi}^{2}(\bar{K}+M(q \bar{\varphi}-f))^{2}}{(M q)^{2}}>q D \Leftrightarrow \\
r \frac{\tilde{\varphi}^{2}(\bar{K}+M(q \bar{\varphi}-f))^{2}}{(M q)^{2}}>q \bar{p} .
\end{gathered}
$$

From the definition of income (12), we know that the left-hand side of the inequality (B.12) is greater then $I$. Because it cannot be that $I<q \bar{p}$ (otherwise there would be no consumption), the inequality holds. This proves that $\frac{\partial D}{\partial \varphi^{*}}>0$.

This finally ensures that $\frac{\partial \mathfrak{f}\left(r, \varphi^{*}\right)}{\partial \varphi^{*}}>0$ and therefore proves the existence and uniqueness of the equilibrium.

Note that this result holds as long as $\tilde{\varphi}, \bar{\varphi}$ and $\int_{\varphi^{*}}^{\infty} \ln (\varphi) \frac{g(\varphi)}{1-G\left(\varphi^{*}\right.} d \varphi$ are defined. This is normally less restrictive than in (Melitz, 2003, p. 1702). The reason for this is that we do not have simple CES preferences, which indicates that normally fewer moments must be defined, which allows for more flexibility (depending on the degree of substitutability). In contrast, the Melitz model contains a finitely defined average productivity and regular price index.

Step 4: Proof of $\frac{\partial f\left(\varphi^{*}, r\right)}{\partial r}>0$.

Finally, we show that $\mathfrak{f}\left(r, \varphi^{*}\right)$, and, therefore, $\varphi^{*}$ are increasing in $r$. 


$$
\begin{aligned}
\frac{\partial \mathfrak{f}\left(\varphi^{*}, r\right)}{\partial r} & =\frac{1}{\left(B+q D-\frac{q}{r}-f\right)^{2}}\left[(B+q D)\left(B+q D-\frac{q}{r}-f\right)\right. \\
& \left.-\left(\frac{q}{r^{2}}\right)(r B+r q D-q)\right] \\
& =(B+q D)\left(B+q D-\frac{q}{r}-f\right)-(B+q D) \frac{q}{r}+\left(\frac{q}{r}\right)^{2} .
\end{aligned}
$$

This implies that the derivative is positive as long as $\left(B+q D-\frac{q}{r}-f\right) \geq \frac{q}{r}$. Suppose that this is not the case. Then, we would have

$$
\begin{aligned}
\frac{q}{r} & \geq B+q D-\frac{q}{r}-f \\
q & \geq \underbrace{r B+r q D-q}_{=c\left(\varphi^{*}\right)(\text { c.p. equation } 2)}-r f \\
r f+q & \geq c\left(\varphi^{*}\right) .
\end{aligned}
$$

However, this contradicts the fact that $\pi\left(\varphi^{*}\right)=c\left(\varphi^{*}\right)-\frac{r}{\varphi^{*}} c\left(\varphi^{*}\right)-r f=0$. This implies that $\varphi^{*}$ is strictly increasing in $r$.

For a better understanding, a graphic analysis of the proof is provided in figure B.4.

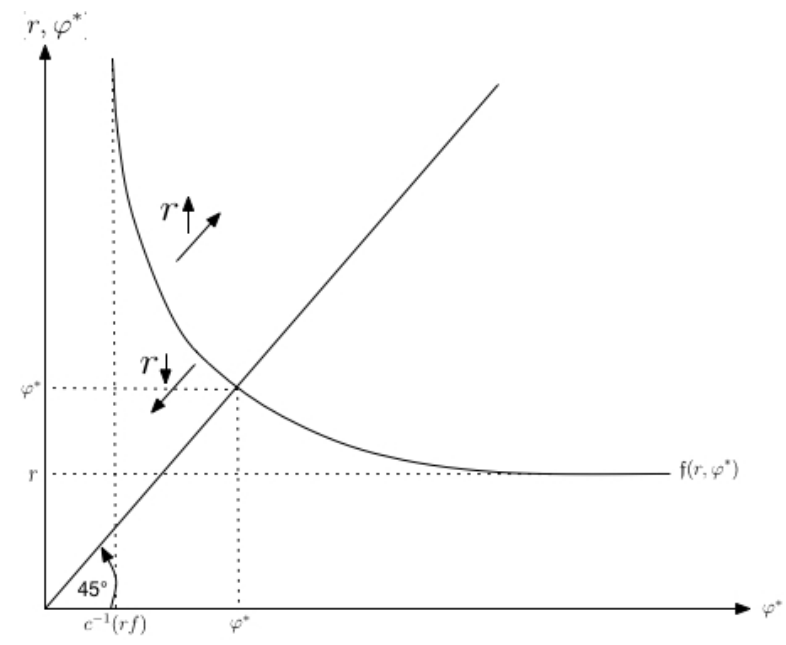

Figure B.4: Existence and uniqueness of the market equilibrium.

Appendix B.2. Proof of Proposition 2.

Proof. We show that there is a welfare-maximizing interest rate $\mathfrak{r}$ by demonstrating that the derivative of the utility function can have positive and negative values. Let us first rewrite the welfare function (13) as 


$$
\begin{aligned}
U & =\frac{M_{0}}{2}\left[\int_{\varphi^{*}}^{\infty} \ln (\varphi) g(\varphi) d \varphi+\left(1-G\left(\varphi^{*}\right)\right) \ln \left(\frac{r B}{r q}+\frac{r D}{r}\right)\right] \\
& =\frac{M_{0}}{2}\left[\int_{\varphi^{*}}^{\infty} \ln (\varphi) g(\varphi) d \varphi+\left(1-G\left(\varphi^{*}\right)\right) \ln \left(\frac{B}{q}+D\right)\right] .
\end{aligned}
$$

The respective derivative is given by

$$
\begin{aligned}
\frac{\partial U}{\partial r} & =\frac{\partial U}{\partial \varphi^{*}} \frac{\partial \varphi^{*}}{\partial r} \\
& =\frac{M_{0}}{2}\left[-g\left(\varphi^{*}\right)\left(\ln \left(\varphi^{*}\right)+\ln \left(\frac{(B+q D)}{q}\right)\right)+\left(1-G\left(\varphi^{*}\right)\right)\left(\frac{\frac{\partial B}{\partial \varphi^{*}}+q \frac{\partial D}{\partial \varphi^{*}}}{B+q D}\right)\right] \frac{\partial \varphi^{*}}{\partial r} \\
& =\frac{M_{0}}{2}[-g\left(\varphi^{*}\right)\left(\ln \left(\varphi^{*}\right)+\ln \left(\frac{(B+q D)}{q}\right)\right) \frac{\partial \varphi^{*}}{\partial r}+\underbrace{\frac{\left(1-G\left(\varphi^{*}\right)\right)}{c\left(\varphi^{*}\right)+q}}_{\geqslant 0} \frac{\partial\left[c\left(\varphi^{*}\right)+q\right]}{\partial r}] .
\end{aligned}
$$

Evaluation occurs for the two extreme values $r \rightarrow \infty$ and $r \rightarrow 0$. From Appendix B.1, we know that $\frac{\partial \varphi^{*}}{\partial r}>0$. Because $\lim _{\varphi^{*} \rightarrow \infty}\left(1-G\left(\varphi^{*}\right)\right)=0$, we can note that

$$
\lim _{\varphi^{*}, r \rightarrow \infty} \frac{\partial \varphi^{*}}{\partial r}=-\infty
$$

Furthermore because $\frac{(B+q D)}{q}$ is finite, and given that $\lim _{\varphi^{*} \rightarrow 0} \ln \left(\varphi^{*}\right)=-\infty$, it follows that

$$
\lim _{\varphi^{*}, r \rightarrow 0} \frac{\partial \varphi^{*}}{\partial r}=\infty
$$

This ensures that the derivative of the welfare function with respect to $r$ consists of a positive and negative part, which proves that there is a welfare-maximizing interest rate, $\mathfrak{r}$. It is clear that more than one interior solution might theoretically exist. However, because computational simulations do not support the relevance of this reservation, it is ignored.

Appendix B.3. Proof of Proposition 4.

Proof. In the following, we show that a stabilizing Taylor rule must be inverse. This is done by demonstrating that $\frac{d \bar{p}}{d r}>0 \wedge \frac{d Y}{d r}>0 \forall r \in[0, \infty)$.

First, the respective derivative for $\bar{p}$ is given by

$$
\frac{d \bar{p}}{d r}=\frac{\partial \bar{p}}{\partial r}+\frac{\partial \bar{p}}{\partial \varphi^{*}} \frac{d \varphi^{*}}{d r}=D+r \frac{\partial D}{\partial \varphi^{*}} \frac{d \varphi^{*}}{d r} .
$$


From Appendix B.1, we know that all parts of this derivate are positive, i.e., $\bar{p}$ is strictly increasing in $r$.

Second, for the derivative of $Y$, let us rewrite the equation (10) for total production as

$$
Y=M_{0}\left(\sqrt{q[B+q D]} \int_{\varphi^{*}}^{\infty} \sqrt{\varphi} g(\varphi) d \varphi-\left(1-G\left(\varphi^{*}\right)\right) q\right) .
$$

The interest rate $r$ has cancelled out, so we can calculate the respective derivative simply by

$$
\begin{aligned}
\frac{\partial Y}{\partial r} & =\frac{\partial Y}{\partial \varphi^{*}} \frac{\partial \varphi^{*}}{\partial r}= \\
& M_{0}\left(\frac{q\left[\frac{\partial B}{\partial \varphi^{*}}+q \frac{\partial D}{\partial \varphi^{*}}\right]}{2 \sqrt{q[B+q D]}} \int_{\varphi^{*}}^{\infty} \sqrt{\varphi} g(\varphi) d \varphi-\sqrt{q[B+q D]} g\left(\varphi^{*}\right) \sqrt{\varphi^{*}}+g\left(\varphi^{*}\right) q\right) \frac{\partial \varphi^{*}}{\partial r}
\end{aligned}
$$

This equation is positive as long as

$$
0.5\left[\frac{\partial B}{\partial \varphi^{*}}+q \frac{\partial D}{\partial \varphi^{*}}\right] \int_{\varphi^{*}}^{\infty} \sqrt{\varphi} g(\varphi) d \varphi \geqslant(B+q D) g\left(\varphi^{*}\right) \sqrt{\varphi^{*}} .
$$

Using equation (B.11), we know that

$$
\frac{\partial B}{\partial \varphi^{*}}+q \frac{\partial D}{\partial \varphi^{*}}=\frac{\left(1+\sqrt{\frac{1}{\tilde{\varphi}^{2}}+\frac{4 B}{q}}\right) \frac{\partial B}{\partial \varphi^{*}}-\left(\tilde{\varphi}+\sqrt{\frac{1}{\tilde{\varphi}^{2}}+\frac{4 B}{q}}\right) \frac{q}{2 \tilde{\varphi}^{3}} \frac{\partial \tilde{\varphi}}{\partial \varphi^{*}}}{\sqrt{\frac{1}{\tilde{\varphi}^{2}}+\frac{4 B}{q}}} .
$$

Given the derivative of $B$, equation (B.10), the second addend ensures that this expression is positive, i.e., the other parts of the equation can be used to show that the condition mentioned is fulfilled. This indicates that the inequality condition (B.21) from above can be reduced to 


$$
\begin{aligned}
& \left(\sqrt{\frac{1}{\tilde{\varphi}^{2}}+\frac{4 B}{q}}\right) 0.5 \overbrace{\left[g\left(\varphi^{*}\right) M_{0} q(\bar{K}+M(q \bar{\varphi}-f))(M q)^{-2}+2 M g\left(\varphi^{*}\right) M_{0} q^{2} \frac{B}{(M q)^{2}}\right]}^{\text {3rd \& 5th addend of eq. (B.10) }} \\
& \int_{\varphi^{*}}^{\infty} \sqrt{\varphi} g(\varphi) d \varphi \\
& \geqslant\left(\sqrt{\frac{1}{\tilde{\varphi}^{2}}+\frac{4 B}{q}}\right)(B+q D) g\left(\varphi^{*}\right) \sqrt{\varphi^{*}} \Leftrightarrow \\
& {\left[(\bar{K}+M(q \bar{\varphi}-f))(M q)^{-1}+B\right] \int_{\varphi^{*}}^{\infty} \sqrt{\varphi} \frac{g(\varphi)}{1-G\left(\varphi^{*}\right)} d \varphi \geqslant(B+q D) \sqrt{\varphi^{*}} \Leftrightarrow} \\
& {\left[r(\bar{K}+M(q \bar{\varphi}-f))(M q)^{-1}+\frac{I}{M}\right] \int_{\varphi^{*}}^{\infty} \sqrt{\varphi} \frac{g(\varphi)}{1-G\left(\varphi^{*}\right)} d \varphi \geqslant\left(\frac{I}{M}+q \bar{p}\right) \sqrt{\varphi^{*}} .}
\end{aligned}
$$

Given that $r(\bar{K}+M(q \bar{\varphi}-f))(M q)^{-1}>I$ (from equation (12)), it also holds that $r(\bar{K}+$ $M(q \bar{\varphi}-f))(M q)^{-1}>q \bar{p}$; otherwise, there would be no consumption. Furthermore, it

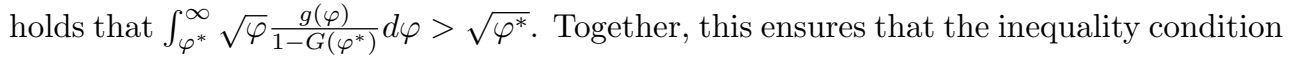
holds and that, therefore, $Y$ is strictly increasing in $r$, which closes the proof.

\section{Appendix C. Optimization by the social planner.}

The social planner solves the optimization problem from equation (14) and considers the impact of the capital stock on the (positive) profits of the firms. The corresponding asset pricing equation therefore becomes $\dot{\mu}(t)=\rho \mu(t)-\frac{\partial I(t)}{\partial K(t)} \mu(t)$. In a steady state, growth rates are zero, i.e., $\dot{\mu}(t)=0 .{ }^{25}$ Using the definition for total income (12) and solving for $r$ yields the steady-state interest rate that a social planer would obtain, denoted by

$$
r_{\text {social }}^{*}:=\frac{\rho M q^{2}}{2 \tilde{\varphi}(\bar{K}+M(q \bar{\varphi}-f))-M q} .
$$

Following the argumentation from section 4, the long-run capital stock determined by the social planner is then given by

$$
\bar{K}=M\left(\sqrt{\frac{q}{r_{\text {social }}^{*} \tilde{\varphi}}\left[\frac{I}{M}+q \bar{p}\right]}-q \bar{\varphi}+f\right) .
$$

The optimization of the social planner entails an equilibrium with a lower interest rate and a higher capital stock, as observed in equations (C.1) and (C.2). This result is not surprising because the social planner considers the effect of an additional unit

${ }^{25}$ It is again assumed that the usual transversality conditions apply. 
of capital on the profits that individuals receive from the firms, i.e., he internalizes the external effect of capital accumulation on profits. The positive effect of capital on profits implies that it is worth saving more than in the decentralized case, which leads to the effects discussed above. However, the qualitative impact of monetary policy as described in sections 5 and 6 also holds with a social planner.

\section{References}

Aghion, P., Farhi, E., Kharroubi, E., 2012. Monetary policy, liquidity, and growth. NBER Working Papers 18072, National Bureau of Economic Research, Inc.

Aghion, P., Howitt, P., 1992. A model of growth through creative destruction. Econometrica 60 (2), 323-351.

Aghion, P., Saint-Paul, G., 1991. On the virtue of bad times: An analysis of the interaction between economic fluctuations and productivity growth. CEPR Discussion Papers 578, CEPR.

Arrow, K., 1962. The economic implications of learning-by-doing. Review of Economic Studies 29, 155173.

Barro, R. J., 1990. Government spending in a simple model of endogenous growth. Journal of Political Economy 98 (5), 103-126.

Barro, R. J., Gordon, D. B., 1983. Rules, discretion and reputation in a model of monetary policy. Journal of Monetary Economics 12 (1), 101-121.

Bernanke, B. S., Gertler, M., 2001. Should central banks respond to movements in asset prices? American Economic Review 91 (2), 253-257.

Bhattacharya, J., Haslag, J., Russell, S., 2005. The role of money in two alternative models: When is the friedman rule optimal, and why? Journal of Monetary Economics 52 (8), 1401-1433.

Bilbiie, F. O., Ghironi, F., Melitz, M., 2007. Monetary policy and business cycles with endogenous entry and product variety. NBER Working Papers 13199, National Bureau of Economic Research, Inc.

Blackburn, K., 1999. Can stabilisation policy reduce long-run growth? The Economic Journal 109 (452), $67-77$.

Blanchard, O. J., 1997. The medium run. Brookings Papers on Economic Activity 2, 89-158.

Broda, C., Weinstein, D., 2007. Product creation and destruction: evidence and price implications. NBER Working Papers 13041, National Bureau of Economic Research, Inc.

Caballero, R. J., Hammour, M. L., 1994. The cleansing effect of recessions. American Economic Review 84 (5), 1350-1368.

Cass, D., 1965. Optimum growth in an aggregative model of capital accumulation. The Review of Economic Studies 32 (3), 233-240.

Chu, A. C., Cozzi, G., 2013. R\&D and Economic Growth in a Cash-in-Advance Economy. Economics Working Paper Series 1308, University of St. Gallen, School of Economics and Political Science.

Darby, M. R., 1975. The financial and tax effects of monetary policy on interest rates. Economic Inquiry $13(2), 266-276$.

Dixit, A., Stiglitz, J., 1977. Monopolistic competition and optimum product diversity. American Economic Review 67, 297-308.

Gali, J., Hammour, M., 1993. Long run effects of business cycles. Working paper, MIT.

Gerlach, S., Moretti, L., 2011. Monetary policy and tips yields before the crisis. CEPR Discussion Papers 8560, CEPR.

Guerrieri, V., Lorenzoni, G., 2011. Credit crises, precautionary savings, and the liquidity trap. Working Paper 17583, National Bureau of Economic Research.

Hall, R., 2011. The long sump. American Economic Review 101 (2), 431-469.

Hansen, B. E., 1997. Approximate asymptotic p values for structuras-change tests. Journal of Business and Economic Statistics 15 (1), 60-67. 
Hopenhayn, H. A., 1992. Exit, selection, and the value of firms. Journal of Economic Dynamics and Control 16 (3-4), 621-653.

Kongsamut, P., Rebelo, S., Xie, D., 2001. Beyond balanced growth. Review of Economic Studies 68 (4), 869-882.

Koopmans, T., 1965. On the concept of optimal economic growth. In: delle Scienze, P. A. (Ed.), The econometric approach to development planning. Amsterdam: North-Holland, pp. 225-287.

Krugman, P., 1998. It's baaack: Japan's slump and the return of the liquidity trap. Brookings Papers on Economic Activity 2, 137-187.

Martin, P., Rogers, C. A., 1997. Stabilization policy, learning-by-doing, and economic growth. Oxford Economic Papers 49 (2), 152-166.

Martin, P., Rogers, C. A., 2000. Long-term growth and short-term economic instability. European Economic Review 44 (2), 359-381.

Melitz, M. J., 2003. The impact of trade on intra-industry reallocations and aggregate industry productivity. Econometrica 71 (6), 1695-1725.

Melitz, M. J., Ottaviano, G. I. P., 2008. Market size, trade, and productivity. Review of Economic Studies 75 (1), 295-316.

Mertens, K., Ravn, M. O., 2011. Leverage and the financial accelerator in a liquidity trap. American Economic Review 101 (3), 413-416.

Mortensen, D. T., Pissarides, C. A., 1994. Job creation and job destruction in the theory of unemployment. Review of Economic Studies 61, 397-415.

Ramey, G., Ramey, V. A., 1995. Cross-country evidence on the link between volatility and growth. American Economic Review 85 (5), 1138 - 1151.

Ramsey, F. P., 1928. A mathematical theory of saving. The Economic Journal 38 (152), 543-559.

Rogoff, K., 1985. The optimal degree of commitment to an intermediate monetary target. The Quarterly Journal of Economics 100 (4), 1169-1189.

Saint-Paul, G., 1993. Productivity growth and the structure of the business cycle. European Economic Review 37 (4), 861-883.

Schmitt-Grohé, S., Uribe, M., 2012. The making of a great contraction with a liquidity trap and a jobless recovery. NBER Working Papers 18544, National Bureau of Economic Research, Inc.

Schumpeter, J. A., 1939. Business cycles: A theoretical, historical and statistical analysis of the capitalist process. Mc-Graw-Hill.

Solow, R. M., 2000. Toward a macroeconomics of the medium run. Journal of Economic Perspectives 14 (1), 151-158.

Werning, I., 2011. Managing a liquidity trap: Monetary and fiscal policy. NBER Working Papers 17344, National Bureau of Economic Research, Inc. 